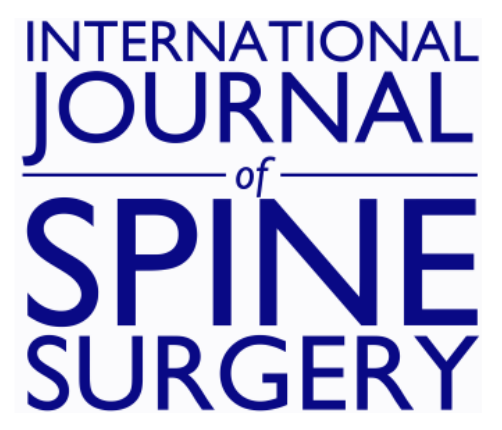

\title{
The Patient-Reported Outcome Measurement Information System (PROMIS) Better Reflects the Impact of Length of Stay and the Occurrence of Complications Within 90 Days Than Legacy Outcome Measures for Lumbar Degenerative Surgery
}

Cole Bortz, Katherine E. Pierce, Haddy Alas, Avery Brown, Dennis Vasquez-Montes, Erik Wang, Christopher G. Varlotta, Dainn Woo, Edem J. Abotsi, Jordan Manning, Ethan W. Ayres, Bassel G. Diebo, Michael C. Gerling, Aaron J. Buckland and Peter G. Passias

Int J Spine Surg 2021, 15 (1) 82-86

doi: https://doi.org/10.14444/8011

http://ijssurgery.com/content/15/1/82

This information is current as of April 25, 2023.

Email Alerts Receive free email-alerts when new articles cite this article. Sign up at: http://ijssurgery.com/alerts 


\title{
The Patient-Reported Outcome Measurement Information System (PROMIS) Better Reflects the Impact of Length of Stay and the Occurrence of Complications Within 90 Days Than Legacy Outcome Measures for Lumbar Degenerative Surgery
}

\author{
COLE BORTZ, BA, ${ }^{1}$ KATHERINE E. PIERCE, BS, ${ }^{1}$ HADDY ALAS, MD,${ }^{1}$ AVERY BROWN, BS, ${ }^{1}$ DENNIS \\ VASQUEZ-MONTES, MS, ${ }^{1}$ ERIK WANG, BA, ${ }^{1}$ CHRISTOPHER G. VARLOTTA, BS, ${ }^{1}$ DAINN WOO, BS, ${ }^{1}$ \\ EDEM J. ABOTSI, BA, ${ }^{1}$ JORDAN MANNING, BA, ${ }^{1}$ ETHAN W. AYRES, MPH, ${ }^{1}$ BASSEL G. DIEBO, MD, ${ }^{2}$ \\ MICHAEL C. GERLING, MD, ${ }^{1}$ AARON J. BUCKLAND, MBBS, FRACS, ${ }^{1}$ PETER G. PASSIAS, MD ${ }^{1}$ \\ ${ }^{1}$ Department of Orthopedics, NYU Langone Orthopedic Hospital, New York, New York, ${ }^{2}$ Department of Orthopedics, SUNY Downstate Medical Center, \\ Brooklyn, New York
}

\begin{abstract}
Background: The Patient-Reported Outcome Measurement Information System (PROMIS) and legacy outcome measures like the Oswestry Disability Index (ODI) have not been compared for their sensitivity in reflecting the impact of perioperative complications and length of stay (LOS) in a surgical thoracolumbar population. The purpose of this study is to assess the strength of PROMIS and ODI scores as they correlate with LOS and complication outcomes of surgical thoracolumbar patients.

Methods: Retrospective cohort study. Included: patients $\geq 18$ years undergoing thoracolumbar surgery with available preoperative and 3-month postoperative ODI and PROMIS scores. Pearson correlation assessed the linear relationships between LOS, complications, and scores for PROMIS (physical function, pain intensity, pain interference) and ODI. Linear regression predicted the relationship between complication incidence and scores for ODI and PROMIS.

Results: Included: 182 patients undergoing thoracolumbar surgery. Common diagnoses were stenosis $(62.1 \%)$, radiculopathy $(48.9 \%)$, and herniated disc $(47.8 \%)$. Overall, $58.3 \%$ of patients underwent fusion, and $50.0 \%$ underwent laminectomy. Patients showed preoperative to postoperative improvement in ODI (50.2 to 39.0), PROMIS physical function (10.9 to 21.4), pain intensity (92.4 to 78.3 ), and pain interference (58.4 to 49.8 , all $P<.001$ ). Mean LOS was $2.7 \pm$ 2.8 days; overall complication rate was $16.5 \%$. Complications were most commonly cardiac, neurologic, or urinary (all $2.2 \%$ ). Whereas preoperative to postoperative changes in ODI did not correlate with LOS, changes in PROMIS pain intensity $(r=0.167, P=.024)$ and physical function $(r=-0.169, P=.023)$ did. Complications did not correlate with changes in ODI or PROMIS score; however, postoperative scores for physical function $(r=-0.205, P=.005)$ and pain interference $(r=0.182, P=.014)$ both showed stronger correlations with complication occurrence than ODI $(r=0.143, P=$ $.055)$. Regression analysis showed postoperative physical function $\left(R^{2}=0.037, P=.005\right)$ and pain interference $\left(R^{2}=0.028\right.$, $P=.014)$ could predict complications; ODI could not.

Conclusions: PROMIS domains of physical function and pain interference better reflected perioperative complications and LOS than the ODI. These results suggest PROMIS may offer more utility as an outcomes assessment instrument.
\end{abstract}

Level of Evidence: 3 .

Lumbar Spine

Keywords: ODI, Oswestry Disability Index, spine, thoracolumbar, thoracic, outcomes, complication, LOS

\section{INTRODUCTION}

Given a health care climate that emphasizes both evidence-based practice and value-based care, providers across the health care spectrum emphasize the need for a valid, reliable, and efficient measure of patient outcomes. In the spine literature, there exist a number of outcome assessment tools, including general health-related quality of life (HRQL) assessments (eg, EuroQol 5Dimensions [EQ-5D], 36-Item Short Form Survey [SF-36]), ${ }^{1,2}$ pain measures (eg, visual analog scale [VAS], numerical 
rating scale $[\mathrm{NRS}]),{ }^{3,4}$ and condition-specific questionnaires (eg, Scoliosis Research Society Outcomes Questionnaire [SRS-22r], Reflux Disease Questionnaire [RDQ] $)^{5,6}$ For spine patients, the Oswestry Disability Index (ODI) is one of the most thoroughly validated outcome assessment tools for low back pain and disability. ${ }^{7-9}$ Multiple studies show the ODI as sufficiently responsive in reflecting the impact of perioperative complications after thoracolumbar surgery, and others have shown significant relationships between ODI score and length of hospital stay. ${ }^{10-12}$

Despite its reliability and consistency, the ODI has several limitations, including an appreciable floor effect, item-masking score bias, cross-contamination score bias, and weighting of items that are unimportant to some patients completing the questionnaire. ${ }^{13,14}$ Additionally, the length of legacy outcome assessments like the ODI may result in substantial administrative burden for both patients and office staff. In contrast to legacy assessments like the ODI, the Patient Reported Outcome Measurement Information System (PROMIS) uses computerized adaptive testing to offer a more efficient means of capturing patient outcomes. ${ }^{15}$ Computer adaptive tests like PROMIS algorithmically select question items based on patient responses, resulting in a shorter (typically 4- to 6-item) assessment of HRQL. ${ }^{16,17}$

PROMIS aims to offer a valid, reliable, and efficient means of capturing spine surgery patient clinical outcomes. To date, few studies have compared PROMIS and legacy outcome measures like the ODI for their sensitivity in reflecting the impact of perioperative complications and length of hospital stay (LOS). To further establish the validity of PROMIS as a reliable tool for assessing patient outcomes, its relationship to complications and LOS - both common measures of surgical outcomes in the spine literature - warrants further investigation. $^{18}$

\section{MATERIALS AND METHODS}

\section{Data Source and Inclusion Criteria}

This is an institutional review board-approved retrospective cohort study of consecutive, patientreported HRQL outcomes collected at a single tertiary teaching hospital from August 2016 to June 2018. All patients included in this analysis were $\geq 18$ years old, underwent thoracolumbar spine surgery, and had ODI and PROMIS scores recorded at both baseline and 3-month postoperative study intervals.

\section{Data Collection and Outcomes Assessments}

Demographic data (age, biologic sex, body mass index) were reviewed from patient medical records at the time of initial presentation and the 3 -month postoperative study interval. Surgical data, including diagnosis, surgical approach, fusion status, and decompression status were reviewed from each patient's surgical note. The following outcome assessments were administered via tablet at the baseline and 3-month postoperative study intervals: PROMIS instruments of pain intensity, pain interference, and physical function, and ODI.

The PROMIS instruments of pain intensity, pain interference, and physical function use a computer adaptive algorithm to assess patient-reported capability, selecting each questionnaire item based on previous item answers. For each PROMIS domain, scores range from 0 to 100 . Higher pain intensity and pain interference scores indicate inferior outcomes, while higher physical function scores indicate superior outcomes.

The ODI has been cited as the gold standard patient-reported outcome instrument for assessing low back pain and disability. ${ }^{19,20}$ ODI scores range from 0 to 100 , with higher scores indicating greater pain and disability.

\section{Statistical Analysis}

Descriptive analyses provided an overview of the overall cohort's demographic and surgical profile. Paired samples $t$ tests assessed differences between preoperative and postoperative HRQL scores. Pearson bivariate correlation assessed the linear relationships between clinical outcomes (including LOS and perioperative complications) and scores for both PROMIS (physical function, pain intensity, pain interference) and ODI. Linear regression predicted the relationship between perioperative complication incidence and 3-month postoperative scores for ODI and PROMIS. Twosided $P$ values less than .05 were considered statistically significant, and all statistical analyses were conducted using SPSS (version 23; IBM Corp., Armonk, NY). 
Table 1. Results of correlation analyses assessing the linear relationships between clinical outcomes (length of stay and complication incidence) and 3month postoperative scores for Patient-Reported Outcome Measurement Information System (PROMIS) and Oswestry Disability Index (ODI).

\begin{tabular}{llrr}
\hline Clinical Outcome & $\begin{array}{c}\text { Health-Related } \\
\text { Quality of Life Assessment }\end{array}$ & \multicolumn{1}{c}{} & $\boldsymbol{P}$ Value \\
\hline Length of stay & ODI & 0.314 & $<.001^{\mathrm{a}}$ \\
& PROMIS pain intensity & 0.237 & $.001^{\mathrm{a}}$ \\
& PROMIS pain interference & 0.174 & $.019^{\mathrm{a}}$ \\
& PROMIS physical function & -0.296 & $<.001^{\mathrm{a}}$ \\
Complication & ODI & 0.143 & .055 \\
incidence & PROMIS pain intensity & 0.137 & .065 \\
& PROMIS pain interference & 0.182 & $.014^{\mathrm{a}}$ \\
& PROMIS physical function & -0.206 & $.005^{\mathrm{a}}$ \\
\hline
\end{tabular}

${ }^{\text {a }}$ Statistically significant, $P<.05$.

\section{RESULTS}

\section{Cohort Demographic and Surgical Overview}

A total of 182 patients met inclusion criteria and underwent thoracolumbar surgery. Mean patient age at baseline was $55.2 \pm 16.1$ years, mean body mass index was $29.5 \pm 6.3 \mathrm{~kg} / \mathrm{m}^{2}$, and $44.5 \%$ of patients were female. Among the most common diagnoses were stenosis $(62.1 \%)$, radiculopathy $(48.9 \%)$, herniated disc $(47.8 \%)$, degenerative spondylolisthesis $(25.3 \%)$, and degenerative disc disease $(22.0 \%)$.

Overall, $58.3 \%$ of patients underwent fusion (mean fusion length $=2.6 \pm 2.9$ levels), and $50.0 \%$ underwent laminectomy. By surgical approach, $82.9 \%$ of cases were posterior only, $0.5 \%$ were anterior only, and $17.6 \%$ were combined. Mean operative time was $208 \pm 136$ minutes, and mean estimated blood loss was $300 \pm 617 \mathrm{cc}$.

\section{Clinical Outcomes}

After surgery, mean LOS was $2.7 \pm 2.8$ days. The overall perioperative complication rate was $16.5 \%$. By type, perioperative complications were most commonly cardiac, neurologic, urinary, or infection (all $2.2 \%$ ), followed by pulmonary $(1.1 \%)$.

The overall cohort showed significant preoperative to 3-month postoperative improvement in all measures of HRQL: ODI $(50.2 \pm 16.1$ to $39.0 \pm$ 19.2, $P<.001)$, PROMIS physical function $(10.9 \pm$ 11.6 to $21.4 \pm 21.3, P<.001)$, pain intensity (92.4 \pm 9.1 to $78.3 \pm 22.3, P<.001)$, and pain interference $(58.4 \pm 5.8$ to $49.8 \pm 8.6, P=.001)$.

\section{PROMIS and ODI Correlations with Outcomes}

Table 1 presents the results of correlation analyses assessing the linear relationships between
Table 2. Results of correlation analyses assessing the linear relationships between clinical outcomes (length of stay and complication incidence) and baseline to 3-month postoperative changes in Patient-Reported Outcome Measurement Information System (PROMIS) and Oswestry Disability Index (ODI) scores.

\begin{tabular}{llrc}
\hline Clinical Outcome & $\begin{array}{c}\text { Health-Related } \\
\text { Quality of Life Assessment }\end{array}$ & $\boldsymbol{R}$ & $\boldsymbol{P}$ Value \\
\hline Length of stay & ODI & 0.100 & .179 \\
& PROMIS pain intensity & 0.167 & $.024^{\mathrm{a}}$ \\
& PROMIS pain interference & 0.078 & .294 \\
& PROMIS physical function & -0.169 & $.023^{\mathrm{a}}$ \\
Complication & ODI & -0.021 & .781 \\
incidence & PROMIS pain intensity & 0.084 & .258 \\
& PROMIS pain interference & 0.127 & .087 \\
& PROMIS physical function & -0.104 & .161 \\
\hline
\end{tabular}

${ }^{\text {a }}$ Statistically significant, $P<.05$.

clinical outcomes and postoperative scores for PROMIS and ODI. All postoperative HRQL instruments correlated with LOS, and of note, 3month postoperative scores for physical function and pain interference both showed stronger correlations with complication occurrence than ODI.

Additionally, whereas preoperative to postoperative changes in ODI score showed no relationship with LOS, changes in PROMIS pain intensity and physical function were both significantly correlated with LOS (Table 2). Complication occurrence was not correlated with preoperative to postoperative changes in ODI or PROMIS scores.

\section{Regression Analysis}

Although there were no significant associations between complication occurrence and postoperative ODI score $\left(R^{2}=0.015, P=.055\right)$, both postoperative PROMIS physical function $\left(R^{2}=0.037, P=\right.$ $.005)$ and pain interference $\left(R^{2}=0.028, P=.014\right)$ were significantly associated with the occurrence of any complication. There were no significant relationships between perioperative complication occurrence and preoperative to postoperative changes in any HRQL instrument used in this study (all $P>$ $.05)$.

LOS also showed weak but statistically significant relationships with all postoperative HRQL instruments: ODI $\left(R^{2}=0.094, P<.001\right)$, PROMIS pain intensity $\left(R^{2}=0.051, P=.001\right)$, pain interference $\left(R^{2}\right.$ $=0.025, P=.019)$, and physical function $\left(R^{2}=\right.$ $0.083, P<.001)$. Only preoperative to postoperative changes in PROMIS pain intensity $\left(R^{2}=0.023, P=\right.$ $.024)$ and physical function $\left(R^{2}=0.023, P=.023\right)$ were associated with LOS; preoperative to postoperative changes in ODI were not $\left(R^{2}=0.005, P=\right.$ $.179)$. 


\section{DISCUSSION}

The PROMIS item banks offer a number of potential advantages over legacy outcome metrics like the ODI, including less item redundancy and lower administrative burden. ${ }^{21}$ Across multiple patient populations, PROMIS scores have outperformed legacy questionnaires in the psychometric properties of coverage, reliability, and dimensionality, suggesting that PROMIS may be an important step forward in the assessment of patient-reported outcomes. ${ }^{17,22}$ Still, it is unclear how PROMIS compares with the ODI in its ability to reflect the impact of perioperative complications and LOS.

Multiple studies across the surgical literature show significant relationships between longer LOS and lower patient-reported satisfaction scores. ${ }^{23-25}$ Similarly, for patients undergoing thoracolumbar surgery, perioperative complication occurrence has also been shown to correlate with inferior legacy patientreported outcome scores. ${ }^{10}$ For PROMIS to be considered a comparable alternative to legacy patient-reported outcome measures, it should demonstrate equal or greater sensitivity in reflecting the impact of complications and LOS. This study shows that the PROMIS domains of physical function and pain interference outperform the ODI in predicting both LOS and perioperative complication occurrence.

Whereas postoperative ODI score showed no relationship to perioperative complication occurrence, our regression analysis showed a statistically significant relationship between complication occurrence and postoperative PROMIS scores. While the strength of this relationship was weak $\left(R^{2}=0.037\right.$ for PROMIS physical function and $R^{2}=0.028$ for pain interference), these results are encouraging, particularly given that, unlike the ODI, PROMIS assessments are not pathology specific. Compared with the ODI, which was designed to specifically for use in populations of patients with low back pain, PROMIS assessments are more general and were designed to measure symptoms and health concepts across a range of chronic conditions. ${ }^{26}$ The PROMIS domains of physical function and pain interference were able to outperform the ODI in a thoracolumbar patient population, further supporting PROMIS's continued use in surgical spine patient populations.

Additionally, although postoperative ODI score was the strongest predictor of LOS, preoperative to postoperative changes in ODI did not reflect LOS. In contrast, inferior preoperative to postoperative changes in both PROMIS pain intensity and physical function were significantly associated with greater LOS. These results indicate that, in our thoracolumbar patient population, the PROMIS instruments of pain intensity and physical function may show better responsiveness - or sensitivity to clinical change - than the ODI. While additional research is necessary to assess the reliability of these relationships, our results suggest PROMIS should be incorporated as a standard measure of HRQL changes after thoracolumbar spine surgery.

While this study aimed to serve as a broad comparison of PROMIS and ODI, our conclusions are limited by the heterogeneity of the included patient population. Patients included in this study presented with a wide range and severity of thoracolumbar diagnoses and as a result likely underwent procedures with differing techniques and levels of surgical invasiveness. Future studies should look to compare PROMIS and ODI instruments in thoracolumbar populations with more homogenous diagnoses. There is a need for further investigation into the use of the scoring method of the PROMIS metric to improve reliability and broad validity of our results. Additionally, as our study only includes patients from a single university-based tertiary care center, the generalizability of our results is certainly limited. Lastly, to better assess the sensitivity of both PROMIS and ODI in reflecting LOS and complication occurrence, future studies should include patients with clinical follow up beyond 3 months postoperative. Despites these limitations, this report is one of the first in the surgical literature to directly compare the clinical utility of PROMIS and ODI in a thoracolumbar patient population.

\section{CONCLUSIONS}

This study shows that, for patients undergoing thoracolumbar spine surgery, PROMIS better reflects the impact of perioperative complication occurrence and LOS than ODI. Given previous reports demonstrating the lower administrative burden of PROMIS, the results of this study suggest PROMIS may offer greater clinical utility in tracking the outcomes of patients undergoing thoracolumbar surgery.

\section{REFERENCES}

1. EuroQol - a new facility for the measurement of healthrelated quality of life. Health Policy. 1990;16(3):199-208.

2. Ware JE, Sherbourne CD. The MOS 36-item short-form 
health survey (SF-36). I. Conceptual framework and item selection. Med Care. 1992;30(6):473-483.

3. Huskisson EC. Measurement of pain. Lancet (London, England). 1974;2(7889):1127-1131.

4. Downie WW, Leatham PA, Rhind VM, et al. Studies with pain rating scales. Ann Rheum Dis. 1978;37(4):378-381.

5. Asher MA, Lai SM, Glattes RC, et al. Refinement of the SRS-22 Health-Related Quality of Life questionnaire Function domain. Spine (Phila Pa 1976). 2006;31(5):593-597.

6. Roland M, Morris R. A study of the natural history of back pain. Part I: development of a reliable and sensitive measure of disability in low-back pain. Spine (Phila Pa 1976). 1983;8(2):141-144.

7. Fairbank JC, Couper J, Davies JB, et al. The Oswestry Low Back Pain Disability Questionnaire. Physiotherapy. 1980;66(8):271-273.

8. Fisher K, Johnston M. Validation of the Oswestry Low Back Pain Disability Questionnaire, its sensitivity as a measure of change following treatment and its relationship with other aspects of the chronic pain experience. Physiother Theory Pract. 1997;13(1):67-80.

9. Fritz JM, Irrgang JJ. A comparison of a modified Oswestry Low Back Pain Disability Questionnaire and the Quebec Back Pain Disability Scale. Phys Ther. 2001;81(2):776-788.

10. Chotai S, Parker SL, Sivaganesan A, et al. Effect of complications within 90 days on patient-reported outcomes 3 months and 12 months following elective surgery for lumbar degenerative disease. Neurosurg Focus. 2015;39(6):E8. doi:10. 3171/2015.8.FOCUS15302

11. Glassman SD, Hamill CL, Bridwell KH, et al. The impact of perioperative complications on clinical outcome in adult deformity surgery. Spine (Phila Pa 1976). 2007;32(24):2764-2770.

12. McGirt MJ, Parker SL, Chotai S, et al. Predictors of extended length of stay, discharge to inpatient rehab, and hospital readmission following elective lumbar spine surgery: introduction of the Carolina-Semmes Grading Scale. J Neurosurg Spine. 2017;27(4):382-390.

13. Patrick DL, Deyo RA, Atlas SJ, et al. Assessing healthrelated quality of life in patients with sciatica. Spine (Phila $\mathrm{Pa}$ 1976). 1995;20(17):1899-1908; discussion 1909.

14. Müller U, Roeder C, Dubs L, et al. Condition-specific outcome measures for low back pain. Part II: scale construction. Eur Spine J. 2004;13(4):314-324.

15. Fidai MS, Saltzman BM, Meta F, et al. Patient-Reported Outcomes Measurement Information System and legacy patient-reported outcome measures in the field of orthopaedics: a systematic review. Arthrosc J Arthrosc Relat Surg. 2018;34(2):605-614.

16. Fries JF, Bruce B, Cella D. The promise of PROMIS: using item response theory to improve assessment of patient-reported outcomes. Clin Exp Rheumatol. 2005;23(5 Suppl 39):S53-S57.

17. Brodke DS, Goz V, Voss MW, et al. PROMIS PF CAT outperforms the ODI and SF-36 physical function domain in spine patients. Spine (Phila Pa 1976). 2017;42(12):921-929.

18. Gruskay JA. Complications and length of stay following spine surgery: analyzing local and national cohorts. 2015. Yale Medicine Thesis Digital Library Web site. http://elischolar. library.yale.edu/ymtdl/1969. Accessed June 12, 2019.

19. Papuga MO, Mesfin A, Molinari R, et al. Correlation of PROMIS physical function and pain CAT instruments with Oswestry Disability Index and Neck Disability Index in spine patients. Spine (Phila Pa 1976). 2016;41(14):1153-1159.
20. Fairbank JC, Pynsent PB. The Oswestry Disability Index. Spine (Phila Pa 1976). 2000;25(22):2940-2952; discussion 2952.

21. Hung M, Stuart AR, Higgins TF, et al. Computerized adaptive testing using the PROMIS physical function item bank reduces test burden with less ceiling effects compared with the short musculoskeletal function assessment in orthopaedic trauma patients. J Orthop Trauma. 2014;28(8):439-443.

22. Hays RD, Spritzer KL, Fries JF, et al. Responsiveness and minimally important difference for the patient-reported outcomes measurement information system (PROMIS) 20-item physical functioning short form in a prospective observational study of rheumatoid arthritis. Ann Rheum Dis. 2015;74(1):104107.

23. Nguyen Thi PL, Briancon S, Empereur F, et al. Factors determining inpatient satisfaction with care. Soc Sci Med. 2002;54(4):493-504.

24. Sahin B, Yilmaz F, Lee K-H. Factors affecting inpatient satisfaction: structural equation modeling. J Med Syst. 2007;31(1):9-16.

25. Tokunaga J, Imanaka Y. Influence of length of stay on patient satisfaction with hospital care in Japan. Int $J$ Qual Health Care. 2002;14(6):493-502.

26. Cella D, Yount S, Rothrock N, et al. The PatientReported Outcomes Measurement Information System (PROMIS): progress of an NIH roadmap cooperative group during its first two years. Med Care. 2007;45(5 Suppl 1):S3-S11.

Disclosures and COI: The authors received no funding for this study and declare that there are no conflicts of interest related to the submitted work. Personal disclosures outside the submitted work: Dr. Michael C. Gerling reports personal consulting fees for Wolf Endoscopic, other financial or material support from CTL Medical, board or committee membership at Cervical Spine Research Society and American Academy of Orthopaedic Surgeons. Dr. Aaron J. Buckland reports personal consulting fees for NuVasive and Stryker. Dr. Peter G. Passias reports personal consulting fees for Medicrea, Spine Wave, Terumo, and Royal Biologics speaking honoraria from Globus Medical and Zimmer, other support from Allosource, and research support from Cervical Spine Research Society.

Corresponding Author: Peter G. Passias, MD, Department of Orthopedics, NYU Langone Orthopedic Hospital, 301 East 17th St, New York, NY 10003. Phone: (516) 357-8777; Fax: (516) 3570087; Email: Peter.Passias@nyumc.org.

Published 26 February 2021

This manuscript is generously published free of charge by ISASS, the International Society for the Advancement of Spine Surgery. Copyright (C 2021 ISASS. To see more or order reprints or permissions, see http://ijssurgery.com. 